\title{
ANÁLISIS DE ÍNDICES EXTREMOS DE TEMPERATURA MÁXIMA DEL GRUPO DE EXPERTOS PARA LA DETECCIÓN DEL CAMBIO CLIMÁTICO E ÍNDICES EN ANDALUCÍA (1961-2014)
}

\author{
Mónica AGUILAR-ALBA ${ }^{1}$, Luis PEDRAZA LUQUE ${ }^{1}$ \\ ${ }^{1}$ Departamento de Geografia Física y Análisis Geográfico Regional, \\ Facultad de Geografia e Historia. Universidad de Sevilla. \\ luispedrazaluque@hotmail.com, malba@us.es
}

\section{RESUMEN}

Se realiza un análisis de índices de extremos térmicos de temperatura máxima para la región de Andalucía, una de las zonas más vulnerables de Europa al cambio climático. Los datos empleados proceden de la base de datos del European Climate Assessment \& Dataset vinculada al ENSEMBLES Project y gestionada por el Koninklijk Nederlands Meteorologisch Instituut. Se han escogido 9 estaciones meteorológicas distribuidas espacialmente de forma representativa por la región para el periodo 1961-2014. Se ha analizado en cada una de ellas el comportamiento de las series de 4 índices del Expert Team on Climate Change Detection and Indices (días cálidos, días de verano, rachas cálidas y máximo mensual de las máximas diarias), disponibles en la página web del ECA\&D (http://www.ecad.eu/download/). Éstos permitirán evaluar muchos aspectos del cambio climático global, el cual incluye cambios en la intensidad, frecuencia, y duración de la temperatura máxima.

Según los test de tendencia de Mann-Kendall y Spearman y t de Student, existen tendencias estadísticamente significativas al incremento de la temperatura máxima asociada en los 4 índices analizados, siendo especialmente destacables los cambios detectados en las estaciones de primavera y verano. Los resultados se comparan con trabajos precedentes para Andalucía a fin de proporcionar una visión conjunta y actualizada de los cambios en las temperaturas máximas en Andalucía.

Palabras clave: Cambio climático, temperatura máxima, índices térmicos, extremos climáticos, análisis de tendencia, Andalucía

\begin{abstract}
Extremes related to maximum temperature indices are analysed for the region of Andalucía, one of the most vulnerable areas in Europe to climate change. Data used have been provided by the European Climate Assessment \& Dataset linked to the ENSEMBLES Project and managed by the Koninklijk Nederlands Instituut meteorologisch. Nine stations representative of the different climates of the region were selected with data for the period 1961-2014. Four indices provided by the Expert Team on Climate Change Detection and Indices (warm days, summer days, warm spurts and monthly maximum daily maximum), available on the website ECA\&D (http://www.ecad.eu/download/) were analysed. These indices were chosen primarily
\end{abstract}


for assessment of the many aspects of a changing global climate which include changes in intensity, frequency and duration of maximum temperature.

Trends in the indices series were computed and tested for statistical significance using Mann-Kendall test, Spearman Rank test and t-Student test. Results showed widespread significant changes in the four indices associated with warming, being noteworthy the increase for the seasons of spring and summer. The results are compared with previous research carried out for Andalusia to provide a general and updated overview of the changes detected in maximum temperatures in Andalusia.

Key words: climate change, maximum temperature, extreme indices, climatic extremes, trend analysis, Andalusia

\section{INTRODUCCIÓN}

El V Informe del Panel Intergubernamental de Cambio Climático (Intergovernmental Panel on Climate Change) destacaba especialmente los cambios observados en eventos de extremos climáticos a escala global y regional, sin precedente en décadas ni milenios atrás (IPCC, 2014). La OMM (2012) afirma que una de las mayores consecuencias de este cambio es el aumento de los eventos meteorológicos extremos. Zarazúa et al. (2014) entre otros coinciden en que no sólo los incrementos en las temperaturas medias, máximas, y mínimas son evidentes a escala global, sino que también se constata un aumento en la frecuencia de estos extremos a escala regional

En concordancia con el IPCC, Fundación Biodiversidad et al. (2013) subraya que en la mayoría de las regiones europeas habrá más episodios relacionados con extremos de altas temperaturas que con los de bajas. El aumento en la temperatura máxima diaria se dará de manera regionalmente uniforme, aunque con diferencias espaciales a destacar, siendo el extremo meridional de la Península Ibérica una de las zonas más vulnerables del sur de Europa, con un incremento en las temperaturas máximas superior a la media global, y más acusado en los meses estivales (Giorgi \& Lionello, 2008; Lionello et al., 2006). Con ello, los impactos de los extremos climáticos están siendo observados tanto en la escala regional como subregional, por esta razón, los estudios regionales sobre tendencias de extremos térmicos son una prioridad para la mayoría de regiones especialmente vulnerables (IPCC, 2007), como sería Andalucía.

Concretamente, los extremos de temperaturas máximas en Andalucía han experimentado un crecimiento que comienza a partir de 1940 en todas las estaciones menos en invierno, en el que se retrasa hasta los años 70. Excepto en otoño, el aumento de las máximas en esta comunidad tiene el carácter de cambio abrupto localizado en la década de los 80, que hacia los 90 ya supera los límites de significación, para el periodo 1961-1990 (Castro-Díez et al., 2007).

Un evento extremo, climatológicamente hablando, sería aquel que está por debajo o que excede de algún umbral, dependiendo del que mejor correspondiese en función de las características climáticas de la región, entre ellos destacaría el percentil 99, 95 o 90 para máximos, y 10 o 5 para mínimos (DeGaetano, 1996; IPCC, 2012).

Existen varios métodos en la evaluación y observación de cambios en los extremos térmicos; por un lado mediante el estudio del comportamiento estadístico de las series originales de datos de temperatura máxima y mínima diarias, y por otro, a 
través de la aplicación de índices climáticos térmicos (Oria, 2012; Fonseca et al., 2015). Dentro de ésta última aproximación se definen diferentes índices a fin de caracterizar tres aspectos en los que se pueden presentar los cambios: frecuencia, duración e intensidad.

Los índices térmicos, empleados en este estudio representan los eventos diarios extremos de temperatura acontecidos por estación del año, o al año, con garantías de cumplir propiedades robustas estadísticamente. En esta línea, numerosos estudios han detectado cambios significativos generalizados en los extremos de temperatura asociados a una tendencia al calentamiento, especialmente para aquellos índices derivados de la temperatura mínima diaria. Destacar que más del 70\% de la superficie terrestre mundial mostró una significativa disminución anual de noches frías y un significativo aumento anual de noches cálidas. Algunas regiones experimentaron un incremento de más del doble en estos índices. Esto implica una tendencia al aumento de la temperatura mínima diaria en todo el mundo. En cuanto a los índices de extremos térmicos máximos, mostraron cambios similares, pero con magnitudes más pequeñas, no dejando de ser trascendente su estudio en base a los efectos y consecuencias que generan (Alexander et al., 2006).

Dada la dificultad de obtener series largas de temperaturas diarias que reúnan los requisitos de calidad y homogeneidad necesarios para este tipo de estudios, se descartó el empleo de series originales homogeneizadas de temperatura máxima diaria optando por seleccionar un subconjunto de índices de extremos de temperatura máxima disponibles en la base de datos E-OBS, gestionada dentro del European Climate Assessment \& Dataset (ECA\&D). Se ha elegido esta variable en lugar de la precipitación o temperatura mínima, por la mayor trascendencia y consecuencias severas para el medio natural y sociedad andaluzas, y en base a que en los últimos años se han registrado periodos de extremos climáticos de índole térmica máxima de las más importantes en décadas (Sousa et al., 2007). Estudios precedentes, aunque escasos en Andalucía, han puesto de manifiesto la relevancia de los cambios en esta variable (Fernández-Montes \& Rodrigo 2012; 2015). Ello, unido a la importancia del análisis de la temperatura máxima en una región ya de por sí cálida, ha contribuido a motivar este trabajo.

El objetivo principal es analizar la evolución y comportamiento de un conjunto de índices climáticos obtenidos en base a registros diarios de temperatura máxima en 9 estaciones de referencia en Andalucía para el periodo 1961-2014, aunque los resultados se presentan en 3 sectores geográficos en los que se han agrupado esos 9 observatorios (tabla 1). El estudio de las tendencias en las series de índices permitirá describir las características particulares de los extremos térmicos máximos en Andalucía, revelando rasgos en cuanto a intensidad, frecuencia y duración, comparables con otros estudios que emplean similares índices de cambio climático definidos por el Grupo para la Detección del Cambio Climático e Índices o Expert Team on Climate Change Detection and Indices (ETCCDI).

\section{DATOS Y MÉTODOS}

La temperatura máxima diaria se considera como una de las variables climáticas, cuyos cambios en su comportamiento y evolución, en mayor medida puedan afectar 
a los recursos territoriales del ámbito de estudio de este trabajo. Por esta razón se han escogido los 4 índices climáticos obtenidos de la temperatura máxima diaria disponibles en la base de datos del ECA\&D, como fuente de datos empleada, de los 11 definidos para dicha variable por el ETCCDI, para las nueve estaciones de medición y periodo de referencia establecido (1961-2014).

En base a la revisión bibliográfica llevada a cabo sobre los estudios climáticos similares precedentes, se ha escogido dicho periodo temporal de referencia 19612014, con el fin de poder comparar posteriormente los resultados con dichos estudios. Igualmente, se escogió este periodo por cuestiones relativas al periodo común de menor número de lagunas de los observatorios elegidos de la red E-OBS. Los observatorios de análisis fueron seleccionados siguiendo principios de representatividad espacial para la región de estudio.

\begin{tabular}{|c|c|c|}
\hline CÓDIGO ECA\&D & ESTACIÓN DE MEDICIÓN & \multirow{2}{*}{ SECTOR GEOGRÁFICO } \\
\hline 3907 & Almería & \multirow{2}{*}{ Sector Oriental } \\
\hline 417 & Granada & \multirow{2}{*}{ Sector Interior } \\
\hline 231 & Málaga Aeropuerto & \multirow{2}{*}{ Sector Occidental } \\
\hline 337 & Córdoba Aeropuerto & \\
\hline 3963 & Morón de la Frontera & \\
\hline 423 & Sevilla San Pablo & \\
\hline 3838 & Rota & \\
\hline 415 & Cádiz & Huelva \\
\hline 3937 & . &
\end{tabular}

Tabla 1: Estaciones meteorológicas seleccionadas de la red E-OBS y sectores geográficos asociados

Los índices fueron elegidos para considerar cambios en la intensidad, frecuencia y duración de los eventos de temperatura. Los índices se pueden dividir en índices de umbral fijo o relativo, basado en percentiles, como es el caso de TX90p (indicador de días cálidos) que muestra aquellos datos en los que la atribución del evento térmico extremo se hace en función de la superación del percentil 90 en la distribución estadística de los datos; de umbral absoluto (SU25 o días de verano), como aquellos en los que cierto número de días superan ciertos umbrales fijos (como es el caso de $25^{\circ} \mathrm{C}$ ), elegidos normalmente como el valor más relevante en términos de mayor impacto causado; de duración, como WSDI, el cual indica la persistencia de la racha cálida (al menos 6 días consecutivos en que la temperatura máxima diaria supera el percentil 90); y por último, en índices absolutos, como el TXx (o temperatura máxima absoluta mensual), que expresa la intensidad del cambio.

En nuestro estudio se evalúa la tendencia decadal por meses de las series de índices de temperatura máxima a través de la aplicación de las tres pruebas mencionadas no paramétricas de los Rangos de Spearman (Haylock et al., 2008; De Lima et al., 2013) y Mann-Kendall (Sneyers, 1975); Yue \& Pilon, 2004), cuantificando la tasa de incremento y su significación estadística mediante la t-Student, mediante el 
software libre AnClim (Stêpánek, 2008). Así, en aquellos casos en que los test no paramétricos de Mann-Kendall y Spearman, para unos niveles de probabilidad del 95\% y $97,5 \%$ respectivamente, detectasen tendencias estadísticamente significativas se ha cuantificado la tendencia mediante regresión lineal $(p=0,05)$ en cada una de las series de índices. Asimismo se analizan los cambios espacio-temporales en la región por decenios, por sectores geográficos (oriental, interior y occidental) para cada una de las estaciones del año (invierno, primavera, verano y otoño).

\section{RESULTADOS}

Tras la aplicación de las pruebas de significación estadística en las series de índices analizadas, se observa que los cambios en la intensidad de los extremos térmicos son más acusados en primavera y verano. En cuanto a cada uno de los índices analizados (véase tabla 2), se observan diferencias importantes tanto en los cambios y tendencias detectadas, así como en cada sector geográfico.

En lo que concierne a la tendencia decadal del indicador del número de días cálidos (TX90p) se detecta en primavera y verano el incremento de alrededor de un día. Del estudio realizado, se puede concluir con que del número de días cálidos (TX90p), destaca especialmente el valor recogido por el sector oriental de +0,94días/década en verano, así como el $+0,84$ días/década en primavera en el sector interior.

\begin{tabular}{|c|c|c|c|c|}
\hline TX90p (días) & INVIERNO & PRIMAVERA & VERANO & OTOÑO \\
\hline Sector Oriental & & & $+0,94$ & $+0,67$ \\
\hline Sector Interior & & $+0,84$ & $+0,81$ & \\
\hline Sector Occidental & & & & \\
\hline WSDI (días) & INVIERNO & PRIMAVERA & VERANO & OTOÑO \\
\hline Sector Oriental & & & & \\
\hline Sector Interior & & $+0,22$ & $+0,37$ & $+0,39$ \\
\hline Sector Occidental & & & $+0,25$ & \\
\hline TXx $\left({ }^{\circ} \mathbf{C}\right)$ & INVIERNO & PRIMAVERA & VERANO & OTOÑO \\
\hline Sector Oriental & $+0,37$ & $+0,62$ & $+0,67$ & \\
\hline Sector Interior & & $+0,54$ & & \\
\hline Sector Occidental & & & & \\
\hline SU25 (días) & INVIERNO & PRIMAVERA & VERANO & OTOÑO \\
\hline Sector Oriental & & & $+1,21$ & $+1,28$ \\
\hline Sector Interior & & $+0,93$ & & \\
\hline Sector Occidental & & & $+1,01$ & \\
\hline
\end{tabular}

Tabla 2: Tendencias significativas observadas para cada indice de extremos térmicos por sectores geográficos en Andalucía (1961-2014), en valor decadal.

La duración de la racha cálida (WSDI) revela una tendencia significativa con diferencias espaciales. Cabe destacar el sector interior occidental revelando una tendencia de $+0,39$ días/década en otoño. En el sector occidental, el verano presentaría 
cambios significativos en torno a $+0,25$ días por década. El verano sería la estación donde se han detectado las tendencias más significativas estadísticamente, siendo las más destacadas las sucedidas en octubre en el sector interior.

En cuanto a la evolución de la temperatura máxima extrema mensual registrada (TXx), concluir con que se detecta una tendencia general al aumento con similar intensidad en toda la región. Destaca el verano como aquella estación del año que registra un mayor incremento por decenio de los extremos cálidos más acusados, en cambio, la primavera revela los cambios más significativos. El sector geográfico oriental recoge el mayor número de estaciones con tendencias significativas estadísticamente significativas (cabe señalar un incremento de $+0,67^{\circ} \mathrm{C} /$ década en verano). No obstante, la primavera recogería las tendencias más acusadas también en el sector interior, con un cambio en la tendencia decadal de estos extremos térmicos de $+0,5^{\circ} \mathrm{C}$. En este caso, tanto el sector interior como el oriental andaluz, destacarían frente al sector litoral occidental, con incrementos muy superiores a $0,5^{\circ} \mathrm{C}$ cada diez años, mayor en el caso del segundo.

En lo que respecta al número de días de verano (SU25), en los que se superan los $25^{\circ} \mathrm{C}$, igualmente existen diferencias espaciales y según la estación del año. En general, el incremento en la frecuencia de los extremos térmicos máximos, es alrededor de 1 día para cada 10 años. El sector más destacado es nuevamente el oriental, con una tendencia decadal superior al día para las respectivas estaciones de verano y otoño. El sector interior destacaría en primavera, siendo el único sector donde se observan un incremento en la frecuencia de los extremos cercano a un día por década. De igual modo ocurre en Andalucía occidental, donde la tendencia observada se circunscribe a la estación de verano, con una tendencia positiva de un día por década.

En general las estaciones ubicadas en el interior y sector oriental revelan las tendencias más acusadas y significativas estadísticamente. Específicamente, teniéndolas como base para la obtención de los resultados aquí expuestos, Almería y Granada, del sector oriental, y Sevilla y Morón de la Frontera, del interior, serían aquellas estaciones en las que se detectan los mayores cambios en estos extremos térmicos. Por el contrario el sector litoral occidental, apuntado por las estaciones de Huelva o Cádiz, apenas muestra cambios significativos en los cuatro índices.

\section{DISCUSIÓN}

Según Castro-Díez et al. (2007), el que la evolución térmica se parezca al promedio global zonal sugiere que las temperaturas peninsulares están en gran medida gobernadas por el balance radiativo, con un papel menor de los fenómenos dinámicos. Según este trabajo se corrobora la tendencia creciente más abrupta en los últimos 30 años $\left(+0,5^{\circ} \mathrm{C} /\right.$ década de media, en verano y primavera en TXx, para los sectores geográficos y estaciones del año que registran cambios). Sin embargo, los resultados aquí presentados no coinciden con el calentamiento que Castro-Díez et al. (2007) asocia al SW andaluz, de $+1^{\circ} \mathrm{C}$ en el periodo de referencia correspondiente.

Los resultados obtenidos del presente trabajo coinciden con los precedentes de Fernández-Montes y Rodrigo $(2015$; 2012) para el sureste peninsular y para la Península Ibérica respectivamente, con un incremento generalizado de las temperaturas máximas, más acusado en la mitad oriental de Andalucía. También existe concordan- 
cia con las acusadas tendencias observadas en primavera y verano frente al resto de estaciones del año así como con las diferencias entre la Andalucía oriental y mediterránea, y la occidental atlántica. En base a esto último, en ambos trabajos de Fernández-Montes y Rodrigo toma especial protagonismo la tendencia positiva que registran los índices con fuerte impacto en la región como son TX90p y WSDI, mayormente en primavera y verano. Comparar las cifras resulta difícil debido al empleo de periodos de referencia y número de estaciones distintos, pero existe una gran concordancia entre los resultados Fernández-Montes y Rodrigo (2012) y el presente trabajo, para las estaciones y sector geográfico señalado. Coincide con que el sector oriental es el que presenta un cambio en los extremos térmicos más acusado $\left(+0,56^{\circ} \mathrm{C} /\right.$ década según ésta referencia, frente a la tendencia de $+0,52^{\circ} \mathrm{C}$ registrada en el presente estudio para todo el extremo oriental andaluz).

Brunet et al. (2007) estimaría una tendencia media en la temperatura máxima de $+0,51^{\circ} \mathrm{C} /$ década en el extremo meridional peninsular, superior a lo estimado para la tendencia térmica global del IV Informe IPCC (IPCC, 2007). Para TX90p dicho estudio señala una tendencia positiva de $+0,83$ días/década y el WSDI registra una tendencia de 0,44días/década. En nuestro caso, destacaría la coincidencia en la tasa de incremento global para la región de Andalucía $\left(+0,46^{\circ} \mathrm{C} /\right.$ década frente al citado $\left.+0,51^{\circ} \mathrm{C}\right)$. En el caso de TX90p, para la región, el presente trabajo revela un incremento de 0,74 días/década, y +0,30días/década en el WSDI. Se tratan por tanto de valores muy próximos unos de los otros entre ambos estudios.

Al igual que sucede con la tendencia significativa observada para el Algarve portugués para los extremos de temperatura máxima en los meses de verano (De Lima et al., 2013), ocurriría con los índices WSDI y SU25 en Andalucía occidental.

Más allá de factores regionales y/o locales, la variabilidad de la temperatura máxima en España está principalmente relacionada con la circulación atmosférica a gran escala. Las tendencias significativas observadas hacia un incremento general de los extremos térmicos máximos parecen estar relacionados con la menor ocurrencia de situaciones ciclónicas sobre la Península Ibérica (Mosmann et al., 2004). En relación al verano, el estudio de Jacobeit et al. (2003) sobre la variación de situaciones dinámicas, en base a datos mensuales de presiones en Europa y la región del Atlántico norte, detecta un incremento en la frecuencia de situaciones anticiclónicas en la segunda mitad del siglo XX, condición que contribuye al ascenso de las temperaturas e indicadores asociados. En un estudio más reciente, Del Río et al. (2012) confirman que el aumento de las temperaturas en el verano se relaciona, entre otras causas, con una mayor frecuencia de las situaciones y condiciones de bloqueo, subsidencia y estabilidad sobre la superficie del mar en las regiones mediterráneas. Ello está en coherencia con la tendencia al incremento más acusada en primavera y verano en ambos sectores geográficos, más significativo en las estaciones orientales. El incremento observado en este trabajo $\left(+0,55^{\circ} \mathrm{C} /\right.$ década $)$ es semejante al detectado por Del Río et al. (2012) de $+0,50^{\circ} \mathrm{C} /$ década. Por lo tanto, parece confirmarse que las condiciones predominantemente anticiclónicas desde 1980 hasta mediados de los años 2000 parecen ser las responsables de los períodos más cálidos, especialmente en primavera (Fernández-Montes \& Rodrigo, 2012; López et al., 2012) y verano. 
Los resultados obtenidos para el periodo de referencia y estaciones de medición seleccionados concuerdan con las previsiones sobre cambio climático elaboradas por la Junta de Andalucía para el escenario A2 de cambio climático para extremos térmicos. La confirmación de estas previsiones pone de manifiesto la necesidad de tomar medidas en la gestión y ordenación del territorio encaminadas a la mitigación y adaptación de la región ante este cambio climático.

\section{REFERENCIAS}

Alexander, L., et al., (2006). Global observed changes in daily climate extremes of temperature and precipitation. Journal of Geophysical Research, 111 (D5), D05109, 1-22. Recuperado de http://doi.org/10.1029/2005JD006290

Brunetti, M., et al., (2004). Temperature, precipitation and extreme events during the last century in Italy. Global and Planetary Change, 40 (1-2), 141-149. Recuperado de http://doi.org/10.1016/S0921-8181(03)00104-8

Castro-Díez, Y., et al., (2007). Cambios Climáticos Observados en la Temperatura y la Precipitación en Andalucía en el Contexto de la Península Ibérica y Hemisférico. En Climate change in Andalusia: trends and environmental consequences (pp. 57-77). Consejería de Medio Ambiente de la Junta de Andalucía, Sevilla. Recuperado de http://www.juntadeandalucia.es/medioambiente/web/Bloques Tematicos/Educacion_Y_Participacion_Ambiental/Educacion_Ambiental/el_ cambio_climatico_en_andalucia/capitulo3.pdf

De Lima, M. I. P., et al., (2013). Recent changes in daily precipitation and surface air temperature extremes in mainland Portugal, in the period 1941-2007. Atmospheric Research, 127, 195-209. Recuperado de http://doi.org/10.1016/j. atmosres.2012.10.001

Del Río, S., Cano Ortiz, A., Herrero, L., \& Penas, A. (2012). Recent trends in mean maximum and minimum air temperatures over Spain (1961-2006). Theoretical and Applied Climatology, 109 (3-4), 605-626. Recuperado de http://doi. org/10.1007/s00704-012-0593-2

DeGaetano, A.T., 1996: Recent trends in extreme maximum and minimum temperature occurrences in the northeastern United States, Journal of Climate, Vol. 9, pp. 1646-1660.

Fernández-Montes, S., \& Rodrigo, F.S. (2012). Trends in seasonal indices of daily temperature extremes in the Iberian Peninsula, 1929-2005. International Journal of Climatology, 32 (15), 2320-2332. Recuperado de http://doi.org/10.1002/ joc.3399

Fernández-Montes, S., \& Rodrigo, F. S. (2015). Trends in surface air temperatures, precipitation and combined indices in the southeastern Iberian Peninsula (19702007). Climate Research, 63 (1), 43-60. Recuperado de http://doi.org/10.3354/ cr01287

Fonseca, D., et al., (2015). Recent trends of extreme temperature indices for the Iberian Peninsula. Physics and Chemistry of the Earth, Parts $A / B / C$, University of Aveiro, Portugal, 1-23. Recuperado de http://doi.org/10.1016/j.pce.2015.12.005 
Fundación Biodiversidad, et al., (2013). Cambio Climático: Bases Físicas. Guía Resumida del Quinto Informe de Evaluación del IPCC, Grupo de Trabajo I. Ministerio de Agricultura, Alimentación y Medio Ambiente, Madrid, 1-47. Recuperado de http://www.magrama.gob.es/es/cambio-climatico/publicaciones/ publicaciones/Guia_resumida_AR5-IPCC_Bases_F\%C3\%ADsicas tcm7311196.pdf

García-Barrón, L. (2007). La evolución climática del Suroeste de la Península Ibérica basada en registros instrumentales. En Climate change in Andalusia: trends and environmental consequences (pp. 81-95). Consejería de Medio Ambiente de la Junta de Andalucía, Sevilla. Recuperado de http://www.juntadeandalucia. es/medioambiente/web/Bloques_Tematicos/Educacion_Y_Participacion Ambiental/Educacion_Ambiental/el_cambio_climatico_en_andalucia/capitulo4. pdf

Giorgi, F., \& Lionello, P. (2008). Climate change projections for the Mediterranean region. Global and Planetary Change, 63 (2-3), 90-104. Recuperado de http:// doi.org/10.1016/j.gloplacha.2007.09.005

Haylock, M. R., et al., (2008). A European daily high-resolution gridded data set of surface temperature and precipitation for 1950-2006. Journal of Geophysical Research, 113 (D20), D20119. Recuperado de http://doi. org/10.1029/2008JD010201

IPCC. (2007). Climate Change 2007: Synthesis Report. Contribution of Working Groups I, II and III to the Fourth Assessment Report of the Intergovernmental Panel on Climate Change. (R. Pachauri \& A. Reisinger, Eds.). Cambridge University Press, Geneva, Switzerland: IPCC. Recuperado de http://www.ipcc. ch/pdf/assessment-report/ar4/syr/ar4_syr_sp.pdf

IPCC. (2012). Managing the Risks of Extreme Events and Disasters to Advance Climate Change Adaptation. A special Report of Working Groups I and II of the Intervogernmental Panel of Climate Change. (C. B. Field, V. Barros, T. F. Stocker, D. Qin, D. J. Dokken, K. L. Ebi,... P. M. Midgley, Eds.). Cambridge University Press, Cambridge, UK and New York, USA: IPCC. Recuperado de http://doi. org/10.1017/CBO9781139177245

IPCC. (2014). Climate Change 2014: Synthesis Report. Contribution of Working Groups I, II and III to the Fifth Assessment Report of the Intergovernmental Panel on Climate Change. (R. K. Pachauri, M. R. Allen, V. R. Barros, J. Broome, W. Cramer, R. Christ,... J. P. van Ypserle, Eds.). Cambridge University Press, Geneva, Switzerland: IPCC. Recuperado de http://ar5-syr.ipcc.ch/ipcc/ipcc/ resources/pdf/IPCC_SynthesisReport.pdf

Jacobeit, J., et al., (2003). Atmospheric circulation variability in the North-AtlanticEuropean área since the mid seventeenth century. Climate Dynamics, 20, 341352.

Junta de Andalucía (2016). Escenarios de cambio climático. Consejería de Medio Ambiente y Ordenación del Territorio, Junta de Andalucía. [Online] Recuperado de: http://www.juntadeandalucia.es/medioambiente/site/portalweb/ menuitem.6ffc $7 f 4 a 4459 \mathrm{~b} 86 \mathrm{a} 1 \mathrm{daa} 5 \mathrm{c} 105510 \mathrm{e} 1 \mathrm{ca} /$ ?vgnextoid=9970c4f9d8a 26310VgnVCM2000000624e50aRCRD\&vgnextchannel=dd5c693aa8465310V gnVCM1000001325e50aRCRD [Fecha de Acceso 1 Jun. 2016]. 
Lionello, P., et al., (2006). Mediterranean Climate Variability. Elsevier. Development in Earth \& Environmental Sciences. Amsterdam, 4, 399-417. Recuperado de https://www.researchgate.net/publication/200472204_Mediterranean_Climate_ Variability_Elsevier

López, J. I., et al., (2012). Tendencias observadas y las proyecciones futuras en la ocurrencia de eventos cálidos invernales en la Cuenca del Ebro. Asociación Española de Climatología. Recuperado de http://digital.csic.es/handle/10261/67289

Mosmann, V., et al., (2004). Detection of statistically significant trends in the summer precipitation of mainland Spain. Atmospheric Research, 70, (1) $43-53$.

Pita, M. F. (2003). El Clima de Andalucía. En López Ontiveros, A. (Eds.) Geografía de Andalucía. Ariel, Barcelona, (pp. 137-174). Recuperado de http://www.upo. es/ghf/giest/documentos/clima_y_territorio/Pita_climaandalucia.pdf

Sneyers, R. (1975). On the statistical analysis of series of observations. Technical Note, 143, WMO-no. 415, Secretariat of World Meteorological Organization, Geneva, Switzerland.

Stêpánek, P. (2008). AnClim - software for time series analysis (for Windows). Department of Geography, Faculty of Sciences, Masaryk University, Brno, 1-12. 1.6 MB. Recuperado de http://www.climahom.eu/AnClim.html.

Sousa, A., et al., (2007). El cambio climático en Andalucía: evolución y consecuencias medioambientales. Consejería de Medio Ambiente de la Junta de Andalucía, Sevilla, 3-348. Recuperado de http://www. juntadeandalucia.es/medioambiente/site/portalweb/menuitem.7e1cf46dd f59bb227a9ebe205510e1 ca/?vgnextoid=370d2bddf65ff110VgnVCM100000 1325 e50aRCRD\&vgnextchannel=a 5 f5 dfde 043 f43 $10 \mathrm{VgnVCM} 100000$ $1325 \mathrm{e} 50 \mathrm{aRCRD}$

OMM. (2012). Reglamento Técnico de la OMM. Documentos Fundamentales, N ${ }^{\circ} 1$ - General, (2), 1-43. Recuperado de http://www.wmo.int/pages/prog/dra/etrp/documents/1083 Manual_on_ETS_es_rev.pdf

Oria, C. (2012). Tendencia Actual de los Indicadores Extremos de Cambio Climático en la Cuenca del rio Mantaro. Technical Note of the Centro de Predicción Numérica, Dirección General de Meteorología Servicio Nacional de Meteorología e Hidrología, Perú, 1-9. Recuperadodehttp://sania.comunidadandina.org/UpLoad/Contenido/9/25/05_indices $\% 20$ de $\% 20$ eventos $\% 20$ extremos.pdf

Yue, S., \& Pilon, P. (2004). A comparison of the power of the $t$ test, Mann-Kendall and bootstrap tests for trend detection. Hydrological Sciences Journal, 49 (1), 21-37. Recuperado de http://doi.org/10.1623/hysj.49.1.21.53996

Zarazúa, P., et al., (2014). Indices of temperature extremes in the South Gulf Coastal Plains in Mexico. Revista mexicana de ciencias agrícolas, Instituto Nacional de Investigaciones Forestales, Agrícolas y Pecuarias (INIFAP), 10, 1843-1857. Recuperado de https://dialnet. unirioja.es/servlet/articulo? codigo $=4$ 